Osamu Sakamoto • Toshiyuki Kitoh - Toshihiro Ohura

Noriaki Ohya $\cdot$ Kazuie Iinuma

\title{
Novel missense mutation (R94S) in the TAZ (G4.5) gene in a Japanese patient with Barth syndrome
}

Received: November 30, 2001 / Accepted: February 9, 2002

\begin{abstract}
Barth syndrome (BTHS) is a rare X-linked disorder characterized by cardiomyopathy, short stature, neutropenia, and 3-methylglutaconic aciduria. Mutations have been identified in the $T A Z(G 4.5)$ gene in patients with BTHS. This article presents a mutation analysis of this gene in a Japanese boy with cardiomyopathy with abnormal mitochondria, cyclic neutropenia, and 3-methylglutaconic aciduria (type 2). The analysis revealed a novel missense mutation (R94S) caused by a single nucleotide substitution (C-to-A) in this patient.
\end{abstract}

Key words Barth syndrome $\cdot T A Z(G 4.5) \cdot$ Cardiomyopathy $\cdot 3$-Methylglutaconic aciduria $\cdot$ Cyclic neutropenia

\section{Introduction}

Barth syndrome (BTHS, MIM 302060) is an X-linked disorder associated with cardiac and skeletal myopathy, short stature, neutropenia, and abnormal mitochondria (Barth et al. 1983). Because this syndrome includes increased urinary excretion of 3-methylglutaconic acid with normal activity of 3-methylglutaconyl coenzyme A (CoA) hydratase, some investigators also use the term "3methylglutaconic aciduria, type 2" (Gibson et al. 1991). Bione et al. (1996) implicated the $T A Z(G 4.5)$ gene at Xq28 as the gene responsible for BTHS. The gene products,

O. Sakamoto $\cdot$ T. Ohura $(\bowtie) \cdot$ K. Iinuma

Department of Pediatrics, Tohoku University School of Medicine,

1-1 Seiryo-machi, Aoba-ku, Sendai 980-8574, Japan

Tel. +81-22-717-7287; Fax +81-22-717-7290

e-mail: tohura@ped.med.tohoku.ac.jp

T. Kitoh

Department of Pediatrics, Shiga Medical Center for Children,

Shiga, Japan

N. Ohya

Department of Community Health Nursing, Faculty of Nursing,

Shiga University of Medical Science, Shiga, Japan termed "taffazzins," show sequence homology with a superfamily of acyltransferases involved in phospholipid metabolism (Neuwald 1997). So far, more than 20 mutations have been reported in the TAZ (G4.5) gene (D'Adamo et al. 1997; Johnston et al. 1997; Sakamoto et al. 2001).

Most patients with BTHS previously reported have been Caucasians. The present article reports a mutation analysis of a Japanese patient with BTHS.

\section{Patients and methods}

Case report

This male patient was born at 36 weeks gestation with a birth weight of $2600 \mathrm{~g}$. A cardiac murmur was pointed out at the age of 2 months. Echocardiography revealed mild hypertrophy of the left ventricle and interventricular septum with normal ejection fraction. At the age of 1 year, the patient underwent cardiac catheterization for a biopsy of the cardiac muscle, and abnormal-shaped mitochondria with dense bodies were revealed on electron microscopy. Serum lactate and pyruvate were mildly elevated, and serum carnitine was within normal range. At the age of 2 years, the patient's body height was $82 \mathrm{~cm}(-2 \mathrm{SD})$. He also showed cyclic neutropenia with an absolute neutrophil count as low as zero. During periods of neutropenia, his fever sometimes rose and he sometimes developed diarrhea. Urinary organic acids showed increased excretion of 3-methylgultaconic acid, 3-methylglutaric acid, and 3hydroxyisovaleric acid. The activity of 3-methylglutaconyl CoA hydratase of cultured fibroblasts was within normal range (subject No. 1 in Gibson et al. 1991). He was diagnosed as 3-methylglutaconic aciduria, type 2 (BTHS). At the last examination (17 years old), his body height was within normal range $(165.7 \mathrm{~cm})$. Despite exercise intolerance with palpitation, echocardiography showed normal ejection fraction without ventricular dilatation or hypertrophy. In association with his cyclic neutropenia, he also sometimes suffered from stomatitis or perianal abscesses. 
His parents gave their informed consent for a genetic analysis. This study was approved by the Ethical Committee of the Tohoku University School of Medicine.

Direct sequencing of the $T A Z(G 4.5)$ gene

Genomic DNA was extracted from leukocytes with the aid of a Sepa Gene Kit (Sanko Jun-yaku, Tokyo, Japan). Polymerase chain reaction (PCR) was used to amplify all 11 exons including the flanking introns. The sequences of primers for exon 3 were BTHS-KS3 (5'-CGAGGTCG ACGGTATCGGCTGTCCCTCATTCCCTG-3') and BTHS-Rev3 (5'-CAGGAAACAGCTATGACATGCCC TGCCTGGACTG-3'). BTHS-KS3 and BTHS-Rev3 had the KS primer sequence (underlined) and M13 reverse sequence (double-lined) at the $5^{\prime}$ ends of the primers, respectively, to facilitate the cycle sequencing reaction. Primer sequences for other exons are available from the authors on request. PCR products were separated on a $3 \%$ agarose gel and purified with a QIAquick Gel Extraction Kit (Promega, Madison, WI, USA). Cycle sequencing was performed using a Thermo Sequenase cycle sequencing kit and an A.L.F. red DNA sequencer (Amersham Pharmacia Biotech, Uppsala, Sweden).

A

Control

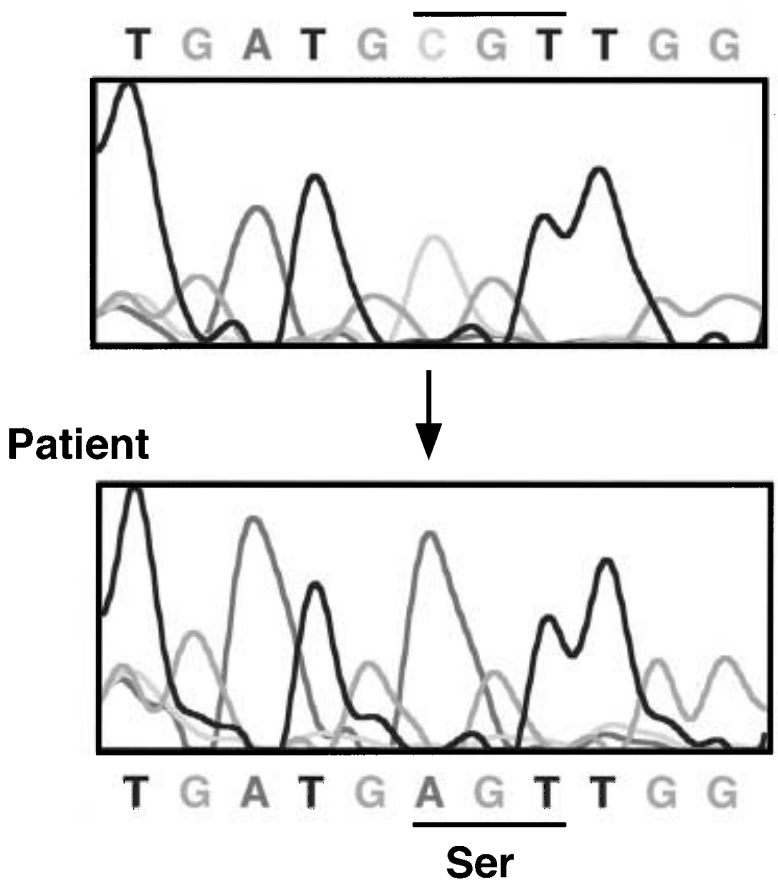

Fig. 1A,B. A Direct sequencing of exon 3 from genomic DNA. The patient showed a C-to-A substitution in a hemizygous pattern. B Detection of the $\mathrm{R} 94 \mathrm{~S}$ mutation by polymerase chain reaction
PCR-restriction fragment length polymorphism for the R94S mutation

PCR amplification was performed for exon 3 as described earlier. PCR products were digested with $S f a$ NI (New England Biolabs, Beverly, MA, USA). Fragments were separated on a $3 \%$ agarose gel and visualized by ethidium bromide staining.

\section{Results and discussion}

In this patient, we found a C-to-A substitution within exon 3 in a hemizygous pattern, resulting in the replacement of arginine with serine (R94S) (Fig. 1A). This R94S mutation abolishes an $S f a$ NI site. PCR products digested with $S f a$ NI showed that the patient was hemizygous and his mother was heterozygous for the R94S mutation (Fig. 1B). The patient's father could not be studied.

Screening for the R94S mutation was performed in DNA samples from 55 healthy volunteers (male: 45 , female: 10 ). All 65 alleles showed wild type (data not shown). The arginine at 94 is conserved in Caenorhabdities elegans and Saccharomyces cerevisiae homologues (D'Adamo et al. 1997), and another patient with a missense mutation at the

B

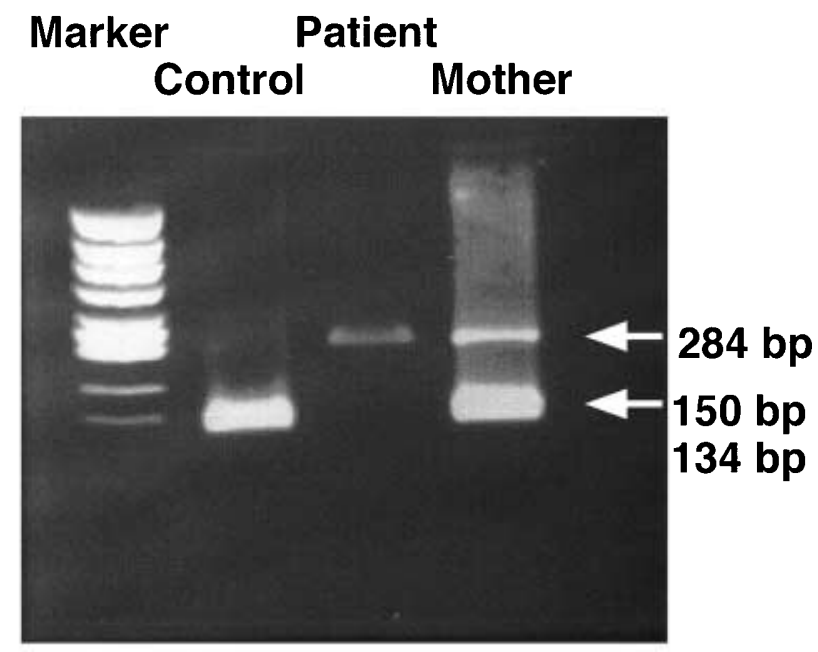

(PCR)-restriction fragment length polymorphism. PCR products digested with $S f a$ NI showed that the patient was hemizygous for the R94S mutation. The mother of the patient was heterozygous 
same position (R94C) has been previously reported (Johnston et al. 1997). These data suggest that R94S is not a common polymorphism, but a disease-causing mutation.

Most patients with BTHS die from cardiac failure or sepsis within 1 year. The present patient had mild cardiac symptoms with normal ejection fraction and contracted no severe infections. Although his body height was $-2 \mathrm{SD}$ at the age of 2 years, he grew to normal height by the age of 17 years. Kelley et al. (1991) reported another affected adult (33 years old) with normal adult height, exercise intolerance, and no history of severe infection. Because there is no correlation between the genotype and phenotype of BTHS (Johnston et al. 1997), some additional factors may be associated with the mild clinical futures of these two patients.

Almost all reported BTHS patients have been from Europe, North America, and Australia. To our knowledge, only two other cases have been reported in Asian populations: one Japanese (Sakamoto et al. 2001; Katsushima et al. 2002) and one Vietnamese (Ichida et al. 2001). However, Cantlay et al. (1999) have questioned whether BTHS is underdiagnosed. We agree that pediatricians and pediatric cardiologists should consider performing metabolic investigations and/or genetic tests for patients with infantile cardiomyopathy.

Acknowledgments The work was supported by a grant from the Ministry of Health and Public Welfare of Japan (Comprehensive Research on Aging and Health).

\section{References}

Barth PG, Scholte JA, Berden JA, Van der Klei-Van Moorsel JM, Luyt-Houwen IEM, Van't Veer-Korthof ET, Van der Harten JJ,
Sobotka-Plojhar MA (1983) An X-linked mitochondrial disease affecting cardiac muscle, skeletal muscle and neutrophil leukocytes. J Neurol Sci 62:327-355

Bione S, D'Adamo P, Maestrini E, Gedeon AK, Bolhuis PA, Toniolo D (1996) A novel X-linked gene, G4.5, is responsible for Barth syndrome. Nat Genet 12:385-389

Cantlay AM, Shokrollabi K, Allen JT, Lunt PW, NewburyEcob RA, Steward CG (1999) Genetic analysis of the G4.5 gene in families with suspected Barth syndrome. J Pediatr 135:311315

D'Adamo P, Fassone L, Gedeon A, Janssen EAM, Bione S, Bolhuis PA, Barth PG, Wilson M, Haan E, Orstavik KH, Patton MA, Green AJ, Zammarchi E, Donati MA, Toniolo D (1997) The X-linked gene $G 4.5$ is responsible for different infantile dilated cardiomyopathies. Am J Hum Genet 61:862-867

Gibson KM, Sherwood WG, Hoffmann GF, Stumpf DA, Dianzani I, Schutgens RBH, Barth PG, Weismann U, Bachmann C, Schrynemackers-Pitance P, Verloes A, Narisawa K, Mino M, Ohya N, Kelley RI (1991) Phenotypic heterogeneity in the syndromes of 3-methylglutaconic aciduria. J Pediatr 118:885-890

Ichida F, Tsubata S, Bowles KR, Haneda N, Uese K, Miyawaki T, Dreyer WJ, Messina J, Li H, Bowles NE, Towbin JA (2001) Novel gene mutations in patients with left ventricular noncompaction or Barth syndrome. Circulation 103:1256-1263

Johnston J, Kelley RI, Feigenbaum A, Cox GF, Iyer GS, Funanage VL, Proujansky R (1997) Mutation characterization and genotypephenotype correlation in Barth syndrome. Am J Hum Genet 61:1053-1058

Katsushima Y, Fujiwara I, Sakamoto O, Ohura T, Miyabayashi S, Ohnuma A, Yamaguchi S, Iinuma K (2002) Normal pituitary function in a Japanese patient with Barth syndrome. Eur J Pediatr 161:67-68

Kelley RI, Cheatham JP, Clark BJ, Nigro MA, Powell BR, Sherwood GW, Sladky JT, Swisher WP (1991) X-linked dilated cardiomyopathy with neutropenia, growth retardation, and 3-methylglutaconic aciduria. J Pediatr 119:738-747

Neuwald AF (1997) Barth syndrome may be due to an acyltransferase deficiency. Curr Biol 7:R465-466

Sakamoto O, Ohura T, Katsushima Y, Fujiwara I, Ogawa E, Miyabayashi S, Iinuma K (2001) A novel intronic mutation of the $T A Z(G 4.5)$ gene in a patient with Barth syndrome: creation of a $5^{\prime}$ splice donor site with variant GC consensus and elongation of the upstream exon. Hum Genet 109:559-563 